\title{
Application of the Numerical Method for the Purposes of the Coal Pillars Failure Zones Estimation with Respect to Excessive Deformation
}

\author{
Inna Ermakova \\ Department «mathematics» \\ T.F. Gorbachev Kuzbass State Technical University) \\ Kemerovo, Russian Federation \\ eia.pm@kuzstu.ru
}

\author{
Natalya Pirieva \\ Department on perspective development \\ OJSC «SUEK-Kuzbass» \\ Leninsk-Kuznetsky, Russian Federation \\ PirievaNN@suek.ru
}

\begin{abstract}
The methodology for calculating the dimensions of failure zones in coal pillars is proposed. The suggested approach is based on the finite element method, taking into consideration excessive deformation of coal seam, and compressive strength of coal. The estimation algorithm is suggested for the failure zones. The correlation between the failure zone length and the seam thickness as well as the mining depth is given herein with respect to various coal compressive strength values.
\end{abstract}

Keywords-coal pillars failure, finite element method, excessive deformation

\section{INTRODUCTION}

The finite element method is widely applied to resolve various geomechanical tasks, including: estimation of the roof-caving increment, detection of the areas with increased abutment pressure, estimation of the failure zones dimensions [1-9]. The results of the failure zones numeric calculations taking into account excessive deformation are presented in this article. The difficulty of such modeling process is as follows: the coal pillar adjacent to the working face is partially destroyed. With increasing distance from the stope into the interior of the pillar, a smooth transformation of the coal mass from a partially destroyed state to an elastic state takes place. An excessive deformation of the coal seam is observed within the zone of destruction. The presence of this zone determines the nature of the abutment pressure curve. The curve reaches its maximum at a certain distance from the pillar edge. The finite element method allows setting different values of deformation and strength characteristics in the selected blocks. Therefore, there is a problem how to set the deformation and strength characteristics of a block of partially destroyed coal in order to achieve a smooth change in stress. To solve this problem, a technique has been developed that makes it possible to simulate the smooth transformation of the edge pillar of the formation from an elastic state to a partially destroyed state, and to obtain a real abutment pressure curve.

\section{METHODS}

The finite element method is applied to estimate of coal pillars stress-strain state. Dependences of the length of the failure zone on the parameters of the problem were obtained by regression and correlation analysis.

\section{RESULTS}

\section{A. Task assignment}

The article considers the solution of the task of a flat stressed-deformed state of host rocks and a coal pillar. Within the rock strata the consideration was given to a section that was normal in relation to the workable seam, and was remote from the mining field boundaries. Within the section considered - a rectangle area has been outlined for the estimation purposes. The area included the following blocks: coal pillar, goaf area with collapsed roof, host rocks of the roof and floor (figure 1).

Once the under stress strength of the pillar was estimated - we had to outline the area in which the destruction took place.

The edges of the pillars are compressed volume-wise. Therefore, it is not seen possible to determine the loss of strength within them, based on the Coulomb-Mohr criteria. A criteria for the compressed rock hardness has been obtained as part of the exercise [10]. The ultimate strength of the leveled seam that experience flat deformation is estimated as follows:

$$
\sigma_{s}^{P}=\gamma H+\frac{\sigma_{s}}{1+v}
$$

In the type: $v$ - transversal deformation factor; $H-$ bedding depth, $\mathrm{m} ; \gamma-$ rock volumetric weight, $\mathrm{N} / \mathrm{m} 3$; $\sigma \mathrm{s}-$ rock uniaxial compressive strength, $\mathrm{N} / \mathrm{m}^{2}$.

As the most powerful stress influencing the seam oyy is vertical - the resulted value appeared to be negative, and therefore the failure parameter was met. The part of the seam where $\left|\boldsymbol{\sigma}_{\boldsymbol{y} \boldsymbol{y}}\right|>\boldsymbol{\sigma}_{\boldsymbol{s}}^{\boldsymbol{P}}$ criteria is met is subject to loss of strength and subsequent partial failure. In other words, it turns into excessive condition, that exceeds the limits.

The strata is likely to change from partially fractured to undisturbed condition heading away from the roadway and getting deeper inside the pillar itself. This zone is likely to experience the linear deformation modulus and the transversal deformation factor changes. But the program being applied for the purposes of finite element method only allows to receive these values as constants. Therefore, we 
input a weaker coal block in the edge of the seam to resolve the contradiction. The block deformation modulus E1 is determined as follows (2):

$$
E_{1}=\frac{\sigma_{s}^{P}-\left(\varepsilon_{0}-\varepsilon_{s}^{P}\right)-M}{\varepsilon_{0}}
$$

In the type: $\boldsymbol{\varepsilon}_{\boldsymbol{s}}^{\boldsymbol{P}}$ - ultimate deformation of the pillar as a result of compression; $\mathbf{E}_{\mathbf{0}}$ - deformation of the pillar's boundary point as a result of compression; $\mathrm{M}$ - excessive deformation modulus, that equals to the coal pillar resilience modulus.

The length of this zone equals to the distance, at which the maximum abutment pressure $\boldsymbol{x}_{\boldsymbol{m}}$ is reached; the vertical dimension equals to the compression value $\boldsymbol{\nu}_{\mathbf{0}}$ available at the edge of the pillar.

\section{B. Estimation algorithm}

The application of the methodology worked out for the purposes of the failure zone dimensions estimation has been considered in the light of the following assumptions: coal pillar width $-300 \mathrm{~m}$, pillar thickness $-2 \mathrm{~m}$, bedding depth $300 \mathrm{~m}$. The following values of the deformation parameters have been used: linear deformation modulus E (Young's modulus) and transversal deformation factor $v$ (Poisson's factor) make $E=1010 \mathrm{MPa}$ and $v=0.2$ for the host rock, as well as $E=109 \mathrm{MPa}$ and $v=0.25$ for the coal. The linear deformation modulus for the rocks that had already failed was set on a pro-rata basis using the weight of the roof above the goaf, and made $\mathrm{E}=\mathrm{H} \cdot 10^{\wedge} 6 \mathrm{~N} / \mathrm{m} 2$, mean time $v=0.3$.

1. An estimation area is outlined. The area includes the following blocks: pillar 1, broken-down rock 2, host rocks 3 (figure 1). All blocks are assigned with respective linear deformation modulus (Young's modulus) values $\mathrm{E}$ and transversal deformation factors (Poisson's factor) $v$, these values are indicated above. All blocks are loaded with the volumetric weight in accordance with the specific gravity of the rocks: $\gamma=13000 \mathrm{~N} / \mathrm{m} 3-$ in the pillar, $\gamma=20000 \mathrm{~N} / \mathrm{m} 3-$ in the zone of broken-down rock, $\gamma=25000 \mathrm{~N} / \mathrm{m} 3$ - in the rocks deposited within the roof and floor.

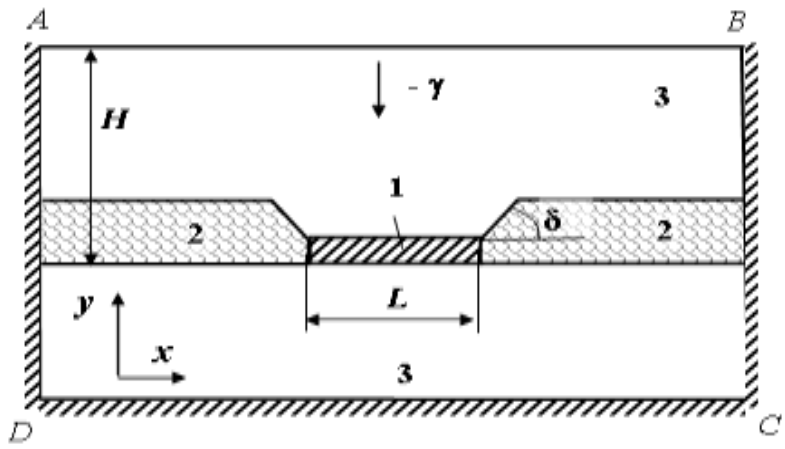

Fig.1. Estimated area

The cut-off parameters were set as follows: there is no load at the upper boundary of the area $A B$ being estimated (daily surface). There is no normal displacement at the lower boundary $\mathrm{CD}$, that is rather remote from the pillar (the boundary is fixed). The horizontal and tangent displacements equal to zero at the vertical boundaries BC and AD.

The height of the zone with collapsed roof made $5 \mathrm{x}$ coal seam thickness and was estimated using the formula: $\boldsymbol{h}_{\boldsymbol{c}}=\boldsymbol{m} /\left(\boldsymbol{k}_{\boldsymbol{c}}-\mathbf{1}\right)$; the fragmentation index of $\mathrm{kc}=1.2$ has been applied. The dipping angle for the rocks of the collapsed floor $\delta$ equals to the natural slope angle, and was assumed at $45^{\circ}$.

2. The seam ultimate strength is estimated using (1):

$$
\sigma_{s}^{P}=25000-300+\frac{10^{7}}{1+0,25}=15,5-10^{6} \mathrm{H} / \mathrm{m}^{2}=15,5 \mathrm{MPa}
$$

3. The vertical stress distribution $\boldsymbol{\sigma}_{\boldsymbol{y} \boldsymbol{y}}$ is estimated for the area being considered.

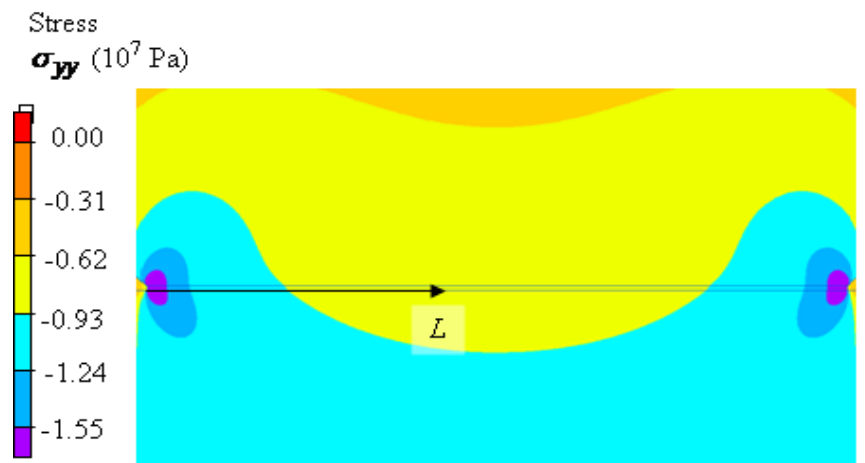

Fig.2. The vertical stress distribution within the strata and the pillar

Maximum compressive stresses are observed in the marginal part of the coal pillar next to the broken-down rock. The zones of destruction are shown in purple, in them $\left|\boldsymbol{\sigma}_{\mathbf{y y}}\right|>\mathbf{1 5 , 5} \mathrm{MPa}$. The resulting picture does not correspond to practical observations in which the maximum of the abutment pressure is at a certain distance from the edge of the pillar. To get the real picture, the following steps are performed.

4. A diagram indicating vertical stress values within the pillar is drawn (figure 3a). The chart is used to determine the length of partially destroyed zone $\boldsymbol{x}_{\boldsymbol{m}}$ (distance to the abutment pressure maximum) using the seam ultimate strength value $\boldsymbol{\sigma}_{\boldsymbol{s}}^{\boldsymbol{p}}$. Given the seam ultimate strength value of $\boldsymbol{\sigma}_{s}^{\boldsymbol{P}}=15,5 \mathrm{MPa}$ (refer to the figure 3b), the length of partially destroyed zone $\boldsymbol{x}_{\boldsymbol{m}}=\mathbf{8 , 5} \mathrm{m}$ - distance to the abutment pressure maximum. a)

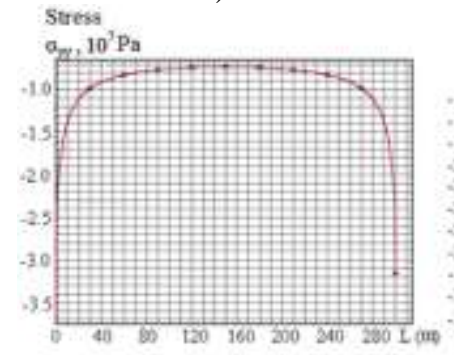

b)

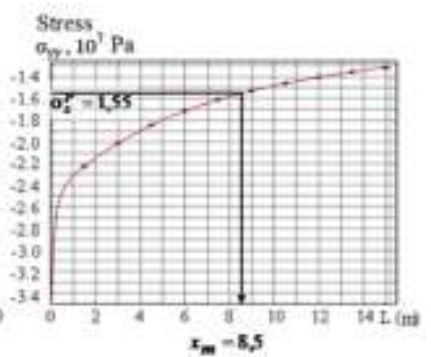

Fig.3. Distribution of the vertical stress values within the pillar, L meters away from its left boundary: a) all the way along the pillar; b) at the left edge of the pillar 
5. The vertical deformation chart is drawn for the edge of the pillar (figure 4). The chart is used to determine the deformation value at the point of the max supporting pressure $\boldsymbol{\varepsilon}_{\boldsymbol{m}}=\boldsymbol{\varepsilon}_{\boldsymbol{s}}^{\boldsymbol{p}}$, as well as the compressive deformation of the pillar $\varepsilon_{0}$ at the boundary point. The results are $\boldsymbol{\varepsilon}_{\boldsymbol{m}}=\boldsymbol{\varepsilon}_{\boldsymbol{s}}^{\boldsymbol{P}}=\mathbf{0 , 0 1 3}$, whereas the compressive deformation of the pillar at the boundary point is $\boldsymbol{\varepsilon}_{\mathbf{0}}=\mathbf{0 , 0 2 7}$.

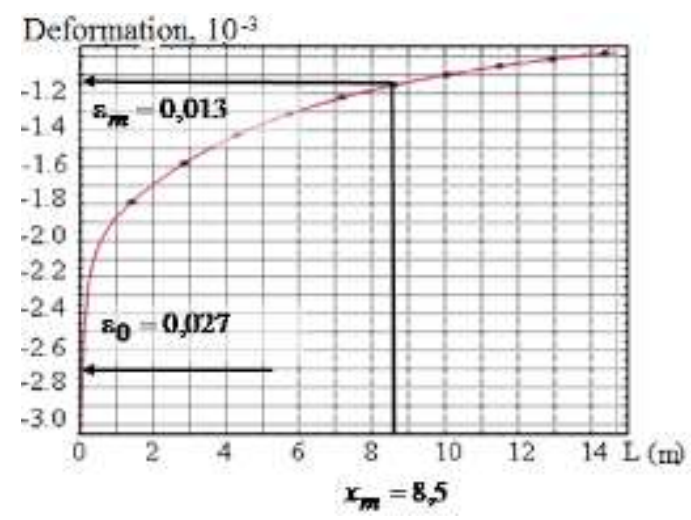

Fig.4. Distribution of the vertical deformation values within the pillar, L meters away from its left boundary

6. The chart is drawn for the vertical displacements of the pillar edge (figure 5). The chart indicates compression value ${ }^{\boldsymbol{\nu}_{0}}$ for the pillar boundary: ${ }^{\boldsymbol{\nu}_{0}}=\mathbf{0 , 2 4 5} \mathrm{m}$.

Displasement, $\mathrm{m}$

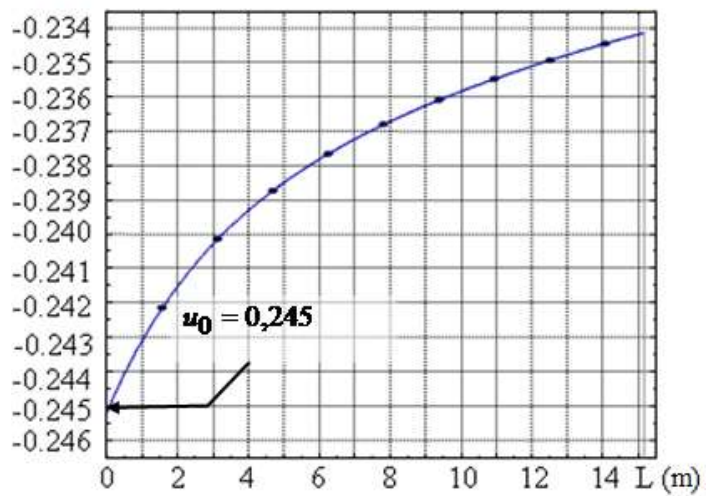

Fig.5. Distribution of the vertical displacement values for the resilient pillar, L meters away from its left boundary

7. The linear deformation modulus for the edge of the seam, which experience excessive condition is estimated using the equation (2):

$$
E_{1}=\frac{15,5 \cdot 10^{6}-(0,027-0,013) \cdot 10^{9}}{0,027}=5,56 \cdot 10^{7}{\mathrm{H} / \mathbf{m}^{2}}_{-}
$$

8. To simulate the smooth transformation of the edge pillar from an elastic state to a partially destroyed state a weaker coal block 4 with deformation modulus $\boldsymbol{E}_{\mathbf{1}}$ is added. The length of this block is equal to the distance to the maximum of the abutment pressure $\boldsymbol{x}_{\boldsymbol{m}}$, whereas the vertical dimension equals to the compression value $\boldsymbol{\nu}_{\mathbf{0}}$ for the edge of the pillar. In this case: the deformation modulus $\boldsymbol{E}_{1}=5,56-10^{7} \mathrm{~N} / \mathrm{m} 2 ; \boldsymbol{x}_{m}=8,5 \mathrm{~m}, \boldsymbol{u}_{0}=0,245 \mathrm{~m}$.

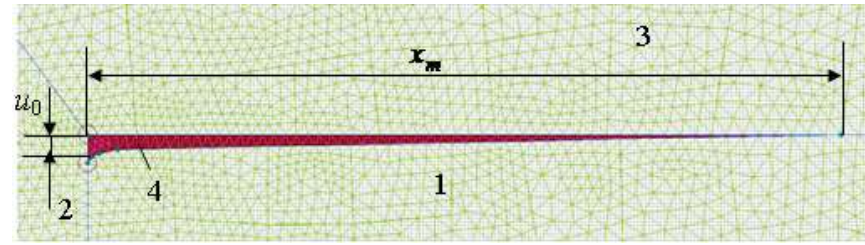

Fig.6. Part of the area that includes the pillar edge: $1-$ coal pillar; 2 - broken-down rock; 3 - host rocks; 4 - weak coal zone

9. The in-pillar vertical stress distribution is assessed once again. The failure zones, in which the vertical stress values exceed the ultimate compressive strength of the seam are outlined: $\left|\boldsymbol{\sigma}_{y \boldsymbol{y}}\right|>\boldsymbol{\sigma}_{s}^{\boldsymbol{P}}$.

The zone of the greatest stresses in the pillar is shown in Fig. 7a with a purple color. The position of this zone has changed in comparison with figure 2 , it has moved deep into the pillar.

Figure $7 \mathrm{~b}$ shows a graph of the values of vertical stresses in the pillar. In contrast to Figure $3 \mathrm{a}$, it shows the maximum of abutment pressure and distance to it $\boldsymbol{x}_{\boldsymbol{m}}$.

a)

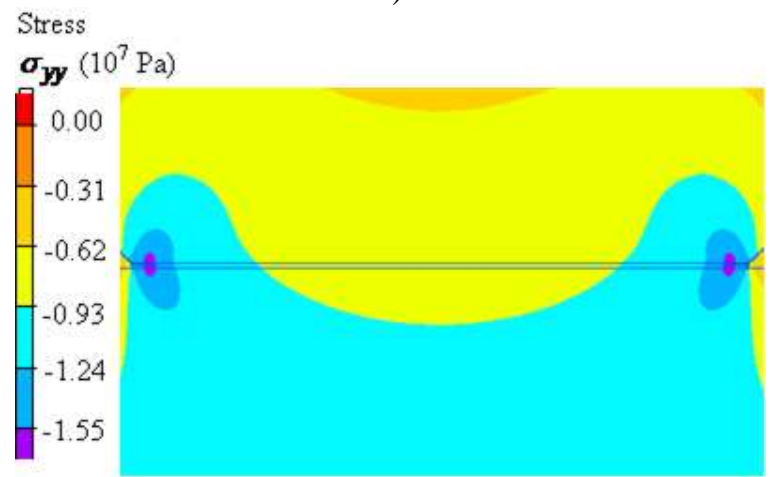

b)

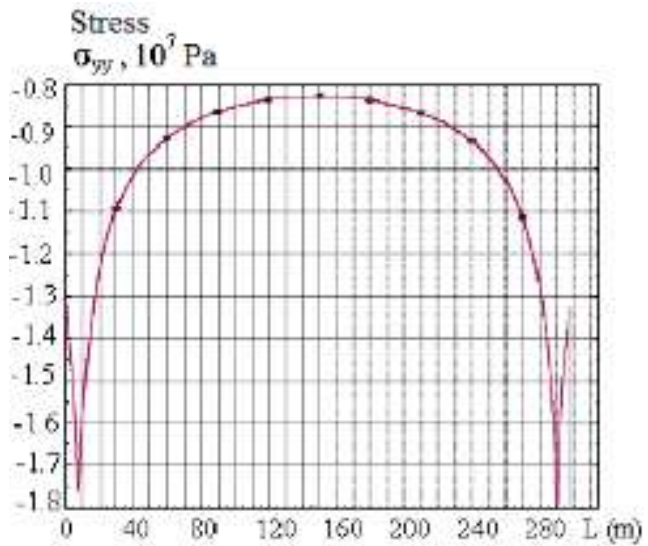

Fig.7. Vertical stress distribution within the rock strata (a) and in the roof of the pillar, that is subject to the excessive deformation (b)

The distribution of the abutment pressure in the pillar is consistent with the existing modern concepts of geomechanical science applied to the extraction of the coal seams.

\section{Practical application}

This methodology has been applied at the mines of Leninsk-Kuznetsky district of Kuzbass to estimate the failure zones within the safety pillars. The longwall mining method 
is used to extract relatively levelled seams within these mines. The method provides for sizable safety pillars to be left between the panels. Therefore the potential of the pillars extraction is there. It is necessary to estimate the dimensions of the failure zones within the pillars to justify the extraction method.

The simulation exercises have been done using the following values:

- mining depth: $H=200,300,400,500 \mathrm{~m}$;

- coal uniaxial compressive strength: ${ }^{\boldsymbol{\sigma}_{s}}=8,10,12 \mathrm{MPa}$;

- coal seam thickness: $m=2,3,4,5 \mathrm{~m}$;

- pillar width: $300 \mathrm{~m}$.

The values of the above-listed factors comply with the parameters to be met in relation to the pillars being considered.

Using a linear regression analysis [11], the dependencies between the failure zone length $\boldsymbol{x}_{\boldsymbol{m}}$, the mining depth $H$, and the thickness of coal seam $m$ were obtained.

$$
\begin{aligned}
& \text { At } \sigma_{s}=8 \mathrm{MPa}: x_{m}=(0,89 m+3,14)-\mathrm{h} H+(-2,77 m-9,83) \\
& \text { At } \sigma_{s}=10 \mathrm{MPa}: x_{m}=(1,32 m+0,72)-\mathrm{h} H+(-5,46 m+0,1) \\
& \text { At } \sigma_{s}=12 \mathrm{MPa}: x_{m}=(1,01 m+1,13)-\mathrm{h} H+(-4,43 m-3,29)
\end{aligned}
$$

\section{CONCLUSIONS}

1) The developed technique for calculating the strained and strength state of the safety pillars allows for the smooth conversion of coal from an elastic state to a partially destroyed one.

2) Such approach allows to adequately describe the distribution of the abutment pressure, and define the dimensions of the failure zones.
3) The researches allowed to establish the regression dependences between the failure zone length, the mining depth, the seam thickness, and the coal compressive strength. The dependences may be used to justify the parameters of the underground coal extraction.

\section{REFERENCES}

[1] Song Wencheng, Zhao Chunbo, Li Guang, Wang Donghui. Research on Simulation and Field Measurement Technology of Floor Mining Failure Depth: Coal in the 21st Century: Mining, Processing and Safety, 2016.

[2] Liu Weitao, Song Wencheng, Wang Jianning. Simulation and Field Measurement Study on Roof Strata Behavior of Fully Mechanized Caving Face: Coal in the 21st Century: Mining, Processing and Safety, 2016.

[3] Ji Yuguo, Wang Xianjun, Zhou Yongpei, Zhang Xiantang. Study on the Distribution Law of Front Abutment Pressure of Long FullyMechanized Working Face in Deep Mine: Coal in the 21st Century: Mining, Processing and Safety, 2016.

[4] Liu Zenghui, Kang Tianhe. Numerical simulation of pillar proper dimension in the entry of mechanized top coal caving face [J]. Ground Pressure and Strata Control, 2005, 22(1):24-26.

[5] Wang Jiachen, Pan Weidong, Li Cheng. 2008. 3D numerical simulation of top coal caving with hard roof in Gequan mine [J]. Journal of Mining \& Safety Engineering, 25(3): 272-276

[6] Griffiths, D. V. and P. A. Lane (1999), "Slope Stability analysis by finite elements." Geotechnique 49(3): 387-403.

[7] ZHAO, Peng; XIE, Ling-Zhi; XIONG, Lun. Numerical simulation of abutment pressure in coal for nonpillar mining, Journal of China Coal Society, Volume 36, Number 12, 15 January 2012, pp. 2029-2034(6).

[8] Chen Zhonghui, Xie Heping. Damage mechanics analysis on the distribution of abutment pressure around a coal face[ J]. Chinese Journal of Rock Mechanics and Engineering, 2000, 19 (4):436-439.

[9] Si Rongjun, Wang Chunqiu, Tan Yunliang. Numerical simulation of abutment pressure distribution laws of working faces [ J]. Rock and Soil Mechanics, 2007, 28(2):351-354.

[10] Gogolin V.A. Rock destruction with volumetric compression: Coal in the 21st Century: Mining, Processing and Safety, 2016: 86-89.

[11] Douglas C. Montgomery, Elizabeth A. Peck, G. Geoffrey Vining. Introduction to Linear Regression Analysis, 5th Edition, 2013: 672 pages. 